\title{
Bidirectional Extraction of Phrases for Expanding Queries in Academic Paper Retrieval
}

\author{
Yuzana Win \\ Graduate School of Engineering \\ Nagasaki University \\ Nagasaki, Japan
}

\author{
Tomonari Masada \\ Graduate School of Engineering \\ Nagasaki University \\ Nagasaki, Japan
}

\begin{abstract}
This paper proposes a new method for query expansion based on bidirectional extraction of phrases as word $n$-grams from research paper titles. The proposed method aims to extract information relevant to users' needs and interests and thus to provide a useful system for technical paper retrieval. The outcome of proposed method are the trigrams as phrases that can be used for query expansion. First, word trigrams are extracted from research paper titles. Second, a co-occurrence graph of the extracted trigrams is constructed. To construct the co-occurrence graph, the direction of edges is considered in two ways: forward and reverse. In the forward and reverse co-occurrence graphs, the trigrams point to other trigrams appearing after and before them in a paper title, respectively. Third, Jaccard similarity is computed between trigrams as the weight of the graph edge. Fourth, the weighted version of PageRank is applied. Consequently, the following two types of phrases can be obtained as the trigrams associated with the higher PageRank scores. The trigrams of the one type, which are obtained from the forward co-occurrence graph, can form a more specific query when users add a technical word or words before them. Those of the other type, obtained from the reverse co-occurrence graph, can form a more specific query when users add a technical word or words after them. The extraction of phrases is evaluated as additional features in the paper title classification task using SVM. The experimental results show that the classification accuracy is improved than the accuracy achieved when the standard TF-IDF text features are only used. Moreover, the trigrams extracted by the proposed method can be utilized to expand query words in research paper retrieval.
\end{abstract}

Keywords-word n-grams; Jaccard similarity; PageRank; TFIDF; query expansion; information retrieval; feature extraction

\section{INTRODUCTION}

In these days, it is an important but complex task to get valuable information by searching the Web. With the rapid increase of information, users often perceive the difficulty of accessing the rich information resource effectively and of obtaining the information associated with their needs accurately. When users want to find the information relevant to their needs, they are required to find appropriate query words or phrases. However, the search results may not be relevant due to the inability of the queries to represent the needs accurately. Especially in academic paper retrieval, in many cases, users also want to find the papers focusing on specific and precise research topics, not general and vague topics. It can be considerably difficult for users to formulate a query for retrieving the papers discussing clear and specific topics. If the query contains only a single word, the search result consists of papers discussing a wide range of topics. That is, while the recall is high, the precision is low. If the query contains too many words, users may get only a limited number of academic papers as a search result. That is, while the precision is high, the recall is low. To overcome the above problems, the solution of this paper is to provide users with help in extracting from a large text set phrases that can be used to expand a less specific query. By expanding queries with the extracted phrases, users may get a search result containing a sufficient number of papers talking about specific research topics.

This paper proposes a new method for extracting important phrases as word $n$-grams from research paper titles. The extracted phrases are expected to be fruitful in query expansion for academic information retrieval. The proposed method is special in the following sense. The method extracts two types of phrases, each of which realizes a different query expansion, i.e., the expansion to the left and the expansion to the right. For example, the proposed method gives "a framework for" and "in sensor networks" as its outcome. The phrase "a framework for" can expand queries like "clustering", "classification", etc., to the left and give more specific queries like "a framework for clustering", "a framework for classification", etc. The phrase "in sensor networks" can expand queries like "clustering", "classification", etc., to the right and give more specific queries like "clustering in sensor networks", "classification in sensor networks", etc.

A brief explanation of the proposed method is given as follows. First, the proposed method extracts word trigrams as phrases that can be used for query expansion from a large number of research paper titles. There are two reasons why we focus on trigrams. The one reason is that, while word $n$-grams will be useful for text analysis, longer $n$-grams may cause data sparseness problem. Because the $n$-grams longer than three may be too long to obtain a sufficient large number of technical papers as a search result. The other is that unigrams and bigrams are too short to make a single word query express a specific and precise topic. Second, the proposed method builds a co-occurrence graph of the extracted trigrams. To construct the co-occurrence graph, the extracted word trigrams are used as nodes and the co-occurrence relations of trigrams appearing in the same paper titles as edges. Here, both the forward and reverse directions of edges are considered. In the forward co-occurrence graph, the trigram points to other trigrams appearing after it in a paper title. In the reverse co-occurrence graph, the trigram points to other trigrams appearing before 
it in a paper title. Third, the proposed method evaluates the Jaccard similarity for all co-occurring pair of trigrams and utilizes the similarity as the edge weight. And fourth, the proposed method applies a weighted version of PageRank on the forward and reverse co-occurrence graphs. As a result, we can get the top-ranked trigrams with reference to PageRank scores. Many of the top-ranked trigrams given from these two co-occurrence graphs can be regarded as important phrases. Details will be explained later.

Our first paper [18] describes a method for exploring technical phrase frames by extracting word $n$-grams. However, this paper introduces a new approach that applies weighted PageRank algorithm on the forward and reverse co-occurrence graphs of trigrams. The distinction between these two types of co-occurrence graphs does not appear in [18]. As a result, the two types of top-ranked trigrams are obtained. The performance of the extracted trigrams are evaluated as additional features in paper title classification using SVM. This evaluation is also not included in [18].

The remainder of this paper is divided into four sections. Section 2 describes the related work. Section 3 explains the proposed method. Section 4 contains the results of the evaluation experiment. The final section concludes the paper with discussion on future work.

\section{RELATED WORK}

The extraction of important word sequences, e.g. keyphrases and key sentences, is relevant to our problem. There are two types of extraction, i.e., supervised [2], [6], [7], [9] and unsupervised methods [1], [3], [4], [8], [10], [11]. Natural language processing techniques [12], [13], [14] have also been used for keyphrase extraction.

Mihalcea [15] proposed an unsupervised method for automatic sentence extraction using graph-based ranking algorithms. The author used a text graph to represent the interconnection of words or other text entities with meaningful relations, ranked the entire sentences in weighted graphs manner, sorted in reversed order of their scores and selected the top ranked sentences for summary. The author evaluated the method in text summarization task. The experimental results show that graph-based ranking algorithms (HITS and PageRank) are useful for sentence extraction when applied to graphs extracted from texts.

Litvak et al. [17] analyzed two graph-based approaches, i.e., unsupervised and supervised ones, which enhance to extract keywords to be used in summarizing documents. The researchers built a graph to represent the co-occurrence in a window of a fixed number of words. They used HITS algorithm to get the top-ranked keyword and identified the keywords in order to generate the summarization. As a result, they argued that if a large number of summarized documents were available then supervised classification was the most accurate to identify the keywords in a document graph. Unless the number of summarized documents are large, unsupervised classification is better to extract the keywords in a graph.

Wan et al. [16] proposed CollabRank, a collaborative approach to single-document keyphrase extraction from multiple documents. They implemented the CollabRank to obtain

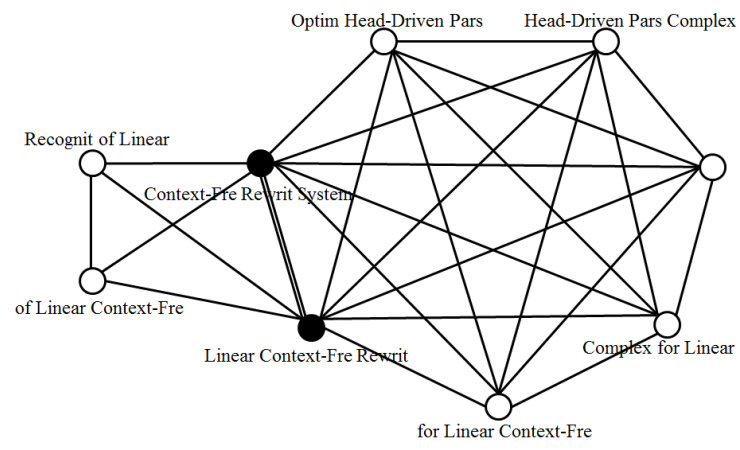

Fig. 1. A small portion of the co-occurrence graph

document clusters by using the clustering algorithm. They used the graph-based ranking algorithm to extract the keyphrases within each document cluster. They built a graph based on all candidate words in the documents of the given cluster and evaluated the candidate phrases in the document based on the scores of the words contained in the phrases. Finally, they chose a few phrases with highest scores as the keyphrases of the document.

Contribution. This paper proposes a method that applies weighted PageRank algorithm on the forward and reverse co-occurrence graphs of trigrams. Consequently, the method can extract two different types of trigrams that can be used for query expansion: 1) Many of the trigrams obtained from the forward co-occurrence graph can form a more specific query when users add a word before them (e.g. "clustering for web search"); 2) Many of the trigrams obtained from the reverse co-occurrence graph can form a more specific query when users add a word after them (e.g. "automatic extraction of clustering"). This kind of bidirectional nature of extraction was not achieved by any of the PageRank-type methods described above.

\section{The Proposed Method}

In this section, the four steps of the proposed method are explained.

\section{A. Word Trigrams}

First, the proposed method extracts trigrams from a large set of research paper titles after applying stemming. For example, the proposed method extracts from the paper title "Recognition of Linear Context-Free Rewriting Systems" the following trigrams: "Recognit of Linear", "of Linear ContextFre", "Linear Context-Fre Rewrit", and "Context-Fre Rewrit System". Word trigrams are extracted by using the natural language toolkit for python (NLTK).

\section{B. Co-occurrence Graph}

The next step of the proposed method is to construct a cooccurrence graph of the extracted trigrams. In order to build the co-occurrence graph, the extracted word trigrams are used as nodes. When two trigrams appear in the same title, they are connected by an edge. Fig. 1 shows a small portion of the co-occurrence graph. This portion is obtained from the 

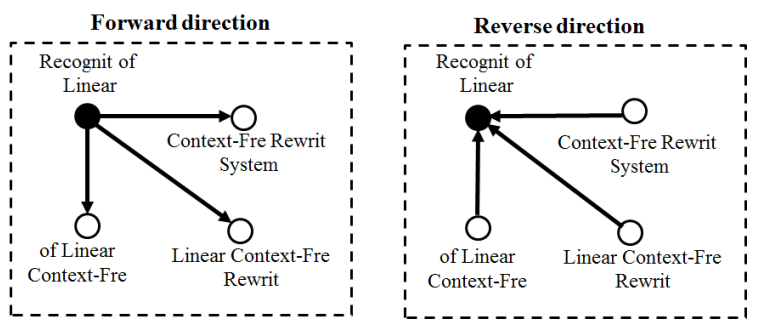

Fig. 2. Co-occurring pairs of trigrams according to forward and reverse directions

following two paper titles: "Recognition of Linear ContextFree Rewriting Systems" and "Optimal Head-Driven Parsing Complexity for Linear Context-Free Rewriting Systems".

Further, the direction of edges is specified according to the order of trigrams. The direction of edges is determined in two ways: forward and reverse directions, as shown in Fig. 2. On the left panel of Fig. 2, the trigram "Recognit of Linear" points to the trigrams "of Linear Context-Free", "Linear ContextFre Rewrit", and "Context-Fre Rewrit System", because the latter three trigrams appear after the trigram "Recognit of Linear" in the paper title "Recognition of Linear ContextFree Rewriting Systems". This direction is called forward direction. In contrast, on the right panel of Fig. 2, the same trigram "Recongnit of Linear" is pointed by the other three trigrams. In this case, each trigram points to the trigrams appearing before it. This direction is called reverse direction. According to the forward and reverse directions of edges, the two co-occurrence graphs, i.e., forward co-occurrence graph and reverse co-occurrence graph, can be constructed.

\section{Jaccard Similarity}

In the third step, the Jaccard similarity is evaluated for all co-occurring pairs of trigrams and the similarity is utilized as the edge weight. Let $\left(t_{1}, t_{2}\right)$ denote a pair of trigrams whose similarity is to be calculated. Let $S\left(t_{i}\right)$ denote the set of paper titles that contain the trigram $t_{i}$. The Jaccard similarity is computed between two trigrams $t_{1}$ and $t_{2}$ as follows:

$$
\operatorname{sim}\left(t_{1}, t_{2}\right)=\frac{\left|S\left(t_{1}\right) \cap S\left(t_{2}\right)\right|}{\left|S\left(t_{1}\right) \cup S\left(t_{2}\right)\right|}
$$

After assigning the Jaccard similarity to each edge, a weighted version of PageRank algorithm is applied. The survey paper [5] analyzed many binary similarity measures. There are two reasons why we compute the Jaccard similarity. The first one is that it is simple to compute. The second one is that the Jaccard similarity is measured with the exclusion of negative matches [5]. In our approach, negative matches are related to the research paper titles where both of the trigrams under consideration do not appear and are not that important.

\section{Weighted PageRank Algorithm}

The last step of the proposed method applies weighted PageRank algorithm on both forward and reverse cooccurrence graphs of the extracted trigrams. Let $\mathrm{P}\left(t_{i}\right)$ denote the PageRank scores of the trigram $t_{i}$. Let $w_{j i}$ denote the weight assigned to the edge connecting the two co-occurring pairs of nodes, $t_{i}$ and $t_{j} . w_{j i}$ is set to the corresponding
TABLE I. DATA SETS

\begin{tabular}{|c|c|}
\hline Fields & Venue \\
\hline \hline NLP & ACL, EACL, COLING, CICLing, NAACL, IJCNLP, EMNLP, NLDB, TSD \\
\hline DM & $\begin{array}{c}\text { SIGMOD, VLDB, PODS, SIGIR, WWW, KDD, ICDE, ISWC, CIDR, } \\
\text { ICDM, ICDT, EDBT, SDM, CIKM, ER, ICIS, SSTD, WebDB, SSDBM, } \\
\text { CAiSE, ECIS, PAKDD }\end{array}$ \\
\hline ALG & STOC, FOCS, ICALP, STACS, ISAAC, MFCS, FSTTCS, FCT, \\
& COCOON, CSR, WoLLIC \\
\hline PRG & $\begin{array}{c}\text { POPL, PLDI, ECOOP, OOPSLA, ISMM, ICLP, ICFP, CGO, ESOP, } \\
\text { FOSSACS, CP, CC, LOPSTR, FLOPS, HOPL, AOSD }\end{array}$ \\
\hline
\end{tabular}

Jaccard similarity. Then the PageRank score of the trigrams is calculated $t_{i}$ by applying the Eq. (2) as below:

$$
P\left(t_{i}\right)=\frac{1-d}{N}+d \times \sum_{t_{j} \in M\left(t_{i}\right)} \frac{w_{j i}}{\sum_{t_{k} \in M\left(t_{j}\right)} w_{j k}} P\left(t_{j}\right)
$$

where $M\left(t_{i}\right)$ denotes the set of nodes which point to $t_{i}$ and $N$ is the total number of extracted trigrams. The parameter $d$ is the damping factor that is usually set to 0.85 . $\sum_{t_{k} \in M\left(t_{j}\right)} w_{j k}$ is the sum of the weights assigned to each neighbor $t_{k}$ in $M\left(t_{j}\right)$. Intuitively, if a node is pointed by many high-scored neighbors, the node may get a high score. However, the proposed method combines the Jaccard similarity and weighted PageRank algorithm. Therefore, if a node is pointed by many high-scored neighbors with large Jaccard similarities, then the node may obtain a high score.

\section{EXPERIMENTAL RESULTS}

\section{A. Evaluation in Text Classification}

The trigrams extracted by the proposed method were evaluated as additional features in the paper title classification task. We used SVM (Support Vector Machine) for classification and checked whether the trigrams extracted by the proposed method improved the classification accuracy.

The proposed method was tested in the binary classification of the paper titles obtained from DBLP (Digital Bibliography $\&$ Library Project) ${ }^{1}$. Each DBLP record included a list of authors, title, conference name or journal name, year, page numbers, etc. Academic conferences were chosen in the four fields: Natural Language Processing (NLP), Data Management (DM), Algorithms and Theory (ALG), and Programming Languages (PRG). We only selected top conferences and used the research paper titles presented in the conferences shown in Table I. As a result, the total number of paper titles contained in NLP, DM, ALG, PRG data sets are 10,666, 27,573, 16,468, and 9,434, respectively. In the preprocessing, stop words were removed and Porter Stemmer ${ }^{2}$ was used to stem words to their root forms.

Classification was conducted on the four data sets, i,e., NLP paper titles, DM paper titles, ALG paper titles and PRG paper titles. From these four data sets, six different pair of data sets were obtained as ALG_PRG, DM_ALG, DM_PRG, NLP_ALG, NLP_DM and NLP_PRG. For each pair, the data was randomly split into $90 \%$ of the paper titles for training and $10 \%$ for testing, and the classification was performed

\footnotetext{
${ }^{1}$ http://www.dblp.com/

${ }^{2}$ http://www.tartarus.org/martin/PorterStemmer/
} 
with SVM. The accuracies were averaged over the ten results obtained from the 10-fold cross-validation.

TF-IDF term weighting was used to compose a feature vector for each paper title based on the formula: $\operatorname{tf}$ idf $(t, d)=$ $\operatorname{tf}(t, d) \times \log (N / \operatorname{df}(t))$, where $\operatorname{tf}(t, d)$ is the frequency of term $t$ in document $d$, and $\operatorname{df}(t)$ is the document frequency of $t$, i.e., the number of documents where $t$ appears. $N$ is the total number of documents in the corpus. In the experiment, the TF in TF-IDF was modified by using the trigrams obtained by the proposed method to improve the classification accuracy. First, we find the trigram having the largest PageRank score in each paper title. For each paper title $d$, the set of the three words of the trigram is denoted having the largest PageRank score by $W(d)$. Then, weight $(t, d)$ is used, defined by Eqs. (3) and (4) in place of $\mathrm{tf}(t, d)$ :

$$
\begin{aligned}
& \text { weight }(t, d) \equiv \alpha \times \operatorname{tf}(t, d)+1 \text { for } t \in W(d) \\
& \text { weight }(t, d) \equiv \alpha \times \operatorname{tf}(t, d) \text { for } t \notin W(d)
\end{aligned}
$$

In Eqs. (3) and (4), $\alpha$ is the term reweighting parameter and is chosen as an integer. All word counts are increased by a factor of $\alpha$ and then the word counts are increased by one only for the words of the trigram having the largest PageRank score (cf. Eq. (3)). For example, we assume that the trigram "probabilist inform flow" has the largest PageRank score among the trigrams appearing in the paper title "Decidability of Parameterized Probabilistic Information Flow" after stemming. Then only the counts of the three words "probabilist", "inform", and "flow" are increased by one after we increase the counts of all words by a factor of $\alpha$. If the trigrams extracted by the proposed method are important in the sense that they are closely related to a particular research topic and thus help discriminating the research topic from other topics, the reweighting described above may improve the classification accuracy.

SVM was trained with linear kernel by setting $C=1.0$ and the classification accuracy was obtained in terms of Area Under the ROC curve (AUC). The term reweighting parameter $\alpha$ was varied from 3 to 27 , and the mean and standard deviation of AUC in the 10-fold cross validation were recorded.

Tables II and III summarize the $p$-values obtained by comparing the standard TF-IDF (i.e., TF-IDF without modification of TF) and the TF-IDF based on the TF modified by Eqs. (3) and (4) in terms of AUC. The $p$-values are obtained in a paired two-sided $t$-test. If the classification accuracy of the proposed method is not as high as the frequency-based method, the $p$ value is assigned with a minus symbol. When the $p$-value is less than 0.05 , we can say that the improvement is statistically significant and thus give the $p$-value in bold in Tables II and III. The results of Table II are given by using the trigrams obtained from the forward co-occurrence graph. On the other hand, the results of Table III are given by using trigrams obtained from the reverse co-occurrence graph. Tables II and III show the term reweighting factor $\alpha$ yielding the best $p$ values on each data set. Only for the two pairs, i.e., DM_PRG and NLP_PRG, in Table III, we could not get a statistically significant improvement. For all remaining cases in Tables II and III, we could get a significantly better accuracy than the standard TF-IDF. Based on these results, it can be said that the classification accuracy is improved by modifying the TF in TF-IDF by the trigrams the proposed method gives. So we claim that the proposed method can extract the features that
TABLE II. $p$-VALUES FOR PAIRED T-TEST ON ROC CURVE AUC (FORWARD)

\begin{tabular}{|l|c|l|c|}
\hline Data sets & Standard TF-IDF & Modified TF-IDF & $p$-value \\
\hline \hline ALG_PRG & 0.942075 & $0.942493(\alpha=3)$ & $\mathbf{0 . 0 0 9}$ \\
\hline DM_ALG & 0.978106 & $0.978225(\alpha=8)$ & $\mathbf{0 . 0 2 1}$ \\
\hline DM_PRG & 0.971507 & $0.971669(\alpha=10)$ & $\mathbf{0 . 0 2 9}$ \\
\hline NLP_ALG & 0.989345 & $0.989452(\alpha=8)$ & $\mathbf{0 . 0 0 3}$ \\
\hline NLP_DM & 0.954356 & $0.954432(\alpha=27)$ & $\mathbf{0 . 0 4 8}$ \\
\hline NLP_PRG & 0.985577 & $0.985633(\alpha=19)$ & $\mathbf{0 . 0 4 7}$ \\
\hline
\end{tabular}

TABLE III. $p$-VALUES FOR PAIRED T-TEST ON ROC CURVE AUC (REVERSE)

\begin{tabular}{|l|c|l|r|}
\hline Data sets & Standard TF-IDF & Modified TF-IDF & $p$-value \\
\hline \hline ALG_PRG & 0.942075 & $0.943616(\alpha=9)$ & $\mathbf{0 . 0 1 3}$ \\
\hline DM_ALG & 0.978106 & $0.978163(\alpha=8)$ & $\mathbf{0 . 0 2 0}$ \\
\hline DM_PRG & 0.971507 & $0.971566(\alpha=23)$ & 0.052 \\
\hline NLP_ALG & 0.989345 & $0.989422(\alpha=12)$ & $\mathbf{0 . 0 1 6}$ \\
\hline NLP_DM & 0.954990 & $0.954883(\alpha=20)$ & -0.053 \\
\hline NLP_PRG & 0.985899 & $0.985959(\alpha=22)$ & $\mathbf{0 . 0 4 1}$ \\
\hline
\end{tabular}

are useful in discriminating different research topics as the trigrams having large PageRank scores.

\section{B. Comparing with Frequency-based Trigram Extraction}

To discuss the special nature of the trigrams extracted by the proposed method, we compared the proposed method with a simple method for the extraction of trigrams, i.e., the frequency-based extraction. In the frequency-based method, the same data sets were used and the same preprocessing were applied as in the proposed method. Then, the number of occurrences, i.e., frequency, were counted for every trigram, and the higher-ranked trigrams based on their frequencies were obtained. The difference between two methods are clarified by displaying examples.

Tables IV and V summarize the trigrams obtained by the frequency-based method and by the proposed method for ALG and DM data sets, respectively. For example, "the complex of" and 381 in the top cell of the left column of Table IV mean that the frequency is 381 for the trigram "the complex of". Moreover, "and relat problem" and $6.05 \times 10^{-4}$ in the top cell of the middle column of Table IV mean that the PageRank is $6.05 \times 10^{-4}$ for the trigram "and relat problem" in the forward co-occurrence graph. Furthermore, "on the complex" and 19.59 $\times 10^{-4}$ in the top cell of the right column of Table IV mean that the PageRank is $19.59 \times 10^{-4}$ for the trigram "on the complex" in the reverse co-occurrence graph.

We can observe that many trigrams obtained from the forward co-occurrence graph can expand queries to the right. For example, the trigram "in web search" can expand the queries like "ranking" and "queries" to give more specific queries like "ranking in web search" and "queries in web search". On the other hand, many trigrams obtained from the reverse co-occurrence graph can expand queries to the left. For example, the trigram "efficient algorithm for" can expand the queries like "computing" and "mining" to give more specific queries like "efficient algorithm for computing" and "efficient algorithm for mining". This is a remarkable feature of the proposed method. In contrast, the frequency-based method 
TABLE IV. TOP-10 (STEMMED) TRIGRAMS OF ALG

\begin{tabular}{|c|c|c|c|c|c|}
\hline \multirow{2}{*}{\multicolumn{2}{|c|}{ Frequency }} & \multicolumn{4}{|c|}{ PageRank } \\
\hline & & \multicolumn{2}{|l|}{ Forward $\left(\times 10^{-4}\right)$} & \multicolumn{2}{|c|}{ Reverse $\left(\times 10^{-4}\right)$} \\
\hline the complex of & 381 & and relat problem & 6.05 & on the complex & 19.59 \\
\hline lower bound for & 259 & in polynomi time & 5.52 & the complex of & 19.23 \\
\hline approxim algorithm for & 225 & in linear time & 5.46 & lower bound on & 8.89 \\
\hline algorithm for the & 209 & and it applic & 4.10 & approxim algorithm for & 8.69 \\
\hline on the complex & 162 & term rewrit system & 3.75 & lower bound for & 8.12 \\
\hline the power of & 103 & in the plane & 3.72 & a note on & 7.20 \\
\hline with applic to & 100 & constraint satisfact problem & 3.24 & bound on the & 7.09 \\
\hline bound on the & 91 & in planar graph & 3.05 & effici algorithm for & 6.84 \\
\hline lower bound on & 81 & of complex class & 2.37 & the power of & 6.13 \\
\hline effici algorithm for & 73 & and their applic & 2.34 & on the power & 5.76 \\
\hline
\end{tabular}

TABLE V. TOP-10 (STEMMED) TRIGRAMS OF DM

\begin{tabular}{|c|c|c|c|c|c|}
\hline \multirow{2}{*}{\multicolumn{2}{|c|}{ Frequency }} & \multicolumn{4}{|c|}{ PageRank } \\
\hline & & \multicolumn{2}{|c|}{ Forward $\left(\times 10^{-4}\right)$} & \multicolumn{2}{|c|}{ Reverse $\left(\times 10^{-4}\right)$} \\
\hline a case studi & 229 & on the web & 4.71 & the impact of & 8.06 \\
\hline the role of & 219 & for inform retriev & 2.91 & the effect of & 6.56 \\
\hline the impact of & 216 & in inform retriev & 2.68 & the role of & 4.41 \\
\hline a framework for & 201 & a case studi & 2.62 & a framework for & 3.39 \\
\hline the effect of & 184 & in web search & 2.50 & a comparison of & 3.04 \\
\hline for inform retriev & 140 & in social network & 2.33 & the influenc of & 2.83 \\
\hline the case of & 140 & an empir studi & 2.03 & a studi of & 2.71 \\
\hline in inform system & 137 & inform retriev system & 2.00 & the use of & 2.06 \\
\hline on the web & 134 & an exploratori studi & 1.94 & effici process of & 1.85 \\
\hline of inform system & 123 & in social media & 1.90 & an analysi of & 1.84 \\
\hline
\end{tabular}

TABLE VI. $\quad p$-VALUES FOR PAIRED T-TEST ON ROC CURVE AUC (FORWARD)

\begin{tabular}{|l|c|c|c|}
\hline Data sets & Frequency-based & Proposed & p-value \\
\hline \hline ALG_PRG & $0.94904(\alpha=5)$ & $0.94928(\alpha=3)$ & 0.058 \\
\hline DM_ALG & $0.98194(\alpha=4)$ & $0.98202(\alpha=2)$ & 0.546 \\
\hline DM_PRG & $0.97613(\alpha=2)$ & $0.97622(\alpha=2)$ & 0.333 \\
\hline NLP_ALG & $0.99140(\alpha=5)$ & $0.99148(\alpha=4)$ & $\mathbf{0 . 0 3 1}$ \\
\hline NLP_DM & $0.96251(\alpha=2)$ & $0.96276(\alpha=2)$ & 0.079 \\
\hline NLP_PRG & $0.98699(\alpha=5)$ & $0.98706(\alpha=4)$ & 0.336 \\
\hline
\end{tabular}

cannot give these two types of trigrams separately, because all trigrams are mixed in the same ranking, as shown in the left columns of Tables IV and V.

Further, we can observe that the frequency-based ranking tends to provide trigrams having a general meaning like "lower bounds for", "the power of", "with applications to", "bounds on the", "lower bounds on", "a case study", "the case of", etc., where the original form is recovered from the root form of each word. In contrast, the proposed method tends to provide trigrams having a specific meaning, e.g. like "in polynomial time ", "in linear time", "in planar graphs", "term rewriting systems", "constraint satisfaction problems", "of complexity classes", "information retrieval system", "an empirical study", etc., with respect to the forward co-occurrence graph. Also with respect to the reverse co-occurrence graph, many trigrams given by the proposed method have at least as specific a meaning as the trigrams given by the frequency-based method. Therefore, it can be said that, at least with respect to the forward co-occurrence graph, the top-ranked trigrams obtained by the proposed method have a more specific meaning than
TABLE VII. $p$-VALUES FOR PAIRED T-TEST ON ROC CURVE AUC (REVERSE)

\begin{tabular}{|l|c|c|r|}
\hline Data sets & Frequency-based & Proposed & p-value \\
\hline \hline ALG_PRG & $0.94245(\alpha=4)$ & $0.94256(\alpha=3)$ & 0.689 \\
\hline DM_ALG & $0.97828(\alpha=5)$ & $0.97822(\alpha=5)$ & -0.384 \\
\hline DM_PRG & $0.97235(\alpha=3)$ & $0.97214(\alpha=3)$ & -0.195 \\
\hline NLP_ALG & $0.98942(\alpha=5)$ & $0.98941(\alpha=5)$ & -0.774 \\
\hline NLP_DM & $0.95450(\alpha=6)$ & $0.95422(\alpha=6)$ & $-\mathbf{0 . 0 2 4}$ \\
\hline NLP_PRG & $0.98563(\alpha=3)$ & $0.98548(\alpha=5)$ & -0.054 \\
\hline
\end{tabular}

those obtained by the frequency-based method.

However, it is possible that the proposed method may degrade the quality of the extracted trigrams by providing them in two separate rankings. Therefore, we compared the proposed method with the frequency-based method also in text classification task described in Section IV-A. We also used SVM (Support Vector Machine) for classification and checked if the trigrams extracted by the proposed method were as useful as the trigrams extracted by the frequency-based method.

To obtain the best classification accuracy in terms of Area Under the ROC curve (AUC), SVM was trained with two different kernels, namely linear kernel by setting $C=1.0$ and rbf (Radial Basis Function) kernel by setting $C=2.0$ and gamma $=2.0$. We selected the term reweighting parameter $\alpha$ yielding the best case from each kernel and recorded the mean and standard deviation of AUC in the 10-fold cross validation.

Tables VI and VII summarize the $p$-values obtained by comparing the frequency-based method and the proposed method based on the TF modified by Eqs. (3) and (4) in 
terms of AUC. The $p$-values are obtained in a paired twosided $t$-test. The $p$-value is assigned with a minus symbol if the classification accuracy of the proposed method is not as high as the frequency-based method. When the $p$-value is less than 0.05 , it can be said that the improvement is statistically significant. The results of Table VI are given by using the trigrams obtained from the forward co-occurrence graph, where SVM is trained by using the rbf kernel. On the other hand, the results of Table VII are given by using the trigrams obtained from the reverse co-occurrence graph, where SVM is trained by using the linear kernel. For all but one case in Tables VI and VII, we could get as good an accuracy as the frequency-based method. We could not get a comparable accuracy only for the NLP_DM data set pair in Table VII. Consequently, the result showed that the proposed method at least could extract as effective trigrams as the frequency-based method. It can be said that the bidirectional nature of the proposed method is an extra gain, which cannot be achieved by the frequency-based method.

\section{A Possible Application: Query Expansion}

Based on the experimental results, it can be said that the trigrams extracted by the proposed method represent technical research topics well. We here discuss how such trigrams can be used in query expansion for information retrieval.

For example, as presented in Fig. 3, the query word "clustering" can be expanded to the right by the trigrams "in sensor networks", "for web search", "for text categorization", etc., which are obtained by the proposed method from the forward co-occurrence graph. These trigrams can be used for the right expansion in this manner, because their first word (i.e., "for" "in", "of", etc.) is a function word that mainly follows a noun. As we discussed in Section IV-B, the trigrams obtained by the proposed method tend to represent a specific meaning, especially with respect to the forward co-occurrence graph. Therefore, we may expect that the search results obtained by the queries expanded in this manner will relate to specific research topics. Fig. 4 gives another example. The query word "clustering" is expanded to the left by the trigrams "a framework for", "automatic extraction of", "efficient algorithm for", etc., which are obtained from the reverse co-occurrence graph. These trigrams can be used for the left expansion, because their last word (i.e., "for", "of", etc.) is a function word that is mainly followed by a noun.

It should be noted that a similar expansion cannot be straightforwardly achieved by the trigrams obtained by the frequency-based method, because the trigrams that can be used for the right expansion and those that can be used for the left expansion are mixed in the same ranking as shown in the left columns of Tables IV and V. However, the proposed method provides two types of trigrams in two different rankings, as shown in the middle and right columns of Tables IV and V.

We here verify how the search results obtained by the expanded queries can focus on more specific research topics. Fig. 5 shows the three types of search results obtained from Google Scholar. Fig. 5(a) gives the search results for the query "clustering". Fig. 5(b) gives the search results obtained by the proposed method from the forward co-occurrence graph. Fig. 5(c) gives the search results obtained by the proposed

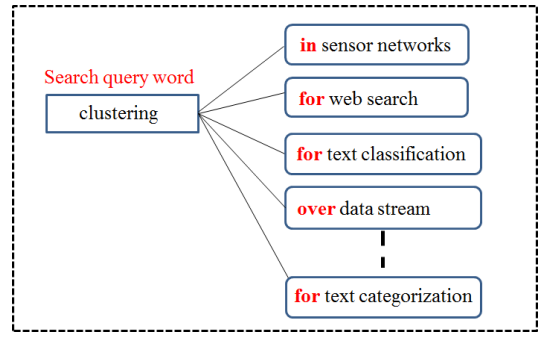

Fig. 3. Example of possible right expansions of the query word 'clustering' by using the trigrams obtained from the forward co-occurrence graph for DM data set

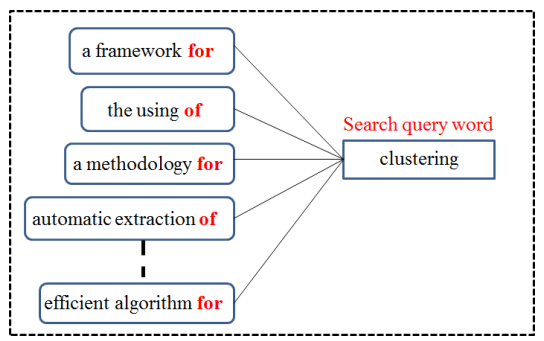

Fig. 4. Example of possible left expansions of the query word 'clustering' by using the trigrams obtained from the reverse co-occurrence graph for DM data set

method from the reverse co-occurrence graph. As presented in Fig. 5(a), we can get the search result having a general meaning when we only input a single query word "clustering". For example, the topics like "Algorithms for clustering data" and "A comparison of document clustering techniques" tend to provide a general meaning consisting of the words like "algorithms" and "techniques". These words tend to represent a wide range of topics. Consequently, the single query word has not exploited users' needs and interests, but users can't get relevant topics when each user has a specific need.

In contrast, when a single query word "clustering" is expanded to the right by the phrase "in sensor networks", we can get the search results focusing on more specific topics as shown in Fig. 5(b). Most of the words or phrases appearing in the search results, e.g., "hybrid", "ad hoc", "hierarchical", and "wireless", have a specific meaning. On the other hand, when a single query word "clustering" is expanded to the left by the phrase "a framework for", we can also get the search results focusing on more specific topics as shown in Fig. 5(c). Some of the words or phrases occurring in the search results, e.g., "data streams", "high dimensional", and "Text and Categorical", represent a specific meaning.

Therefore, it is found that the query expansion, i.e., the expansion to the left and the expansion to the right, can give the search results relating to specific topics. Further, we can get two different types of search results due to the bidirectional nature of the proposed method. These results are more specific when we expand query words than when we only use a single query word. We can observe that the proposed method works as a new query expansion scheme more oriented toward actual user needs and interests for informational retrieval.

\section{Conclusion}

In this paper, we proposed a new method for query expansion based on bidirectional extraction of phrases. The proposed 


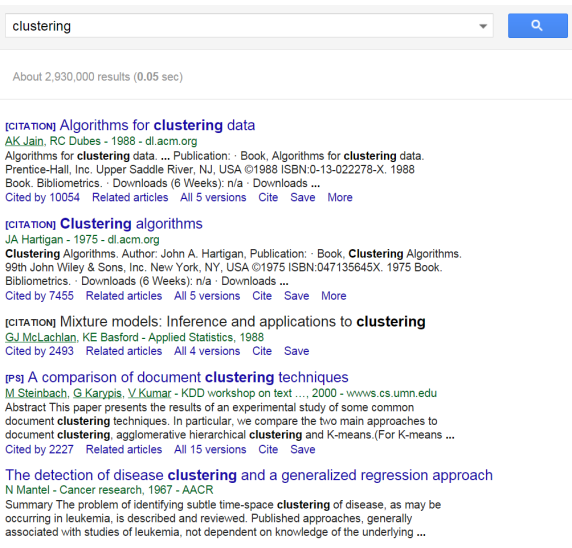

(a)

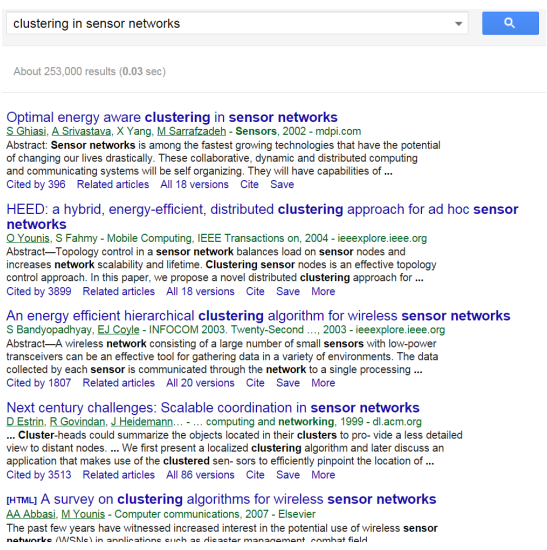

(b)

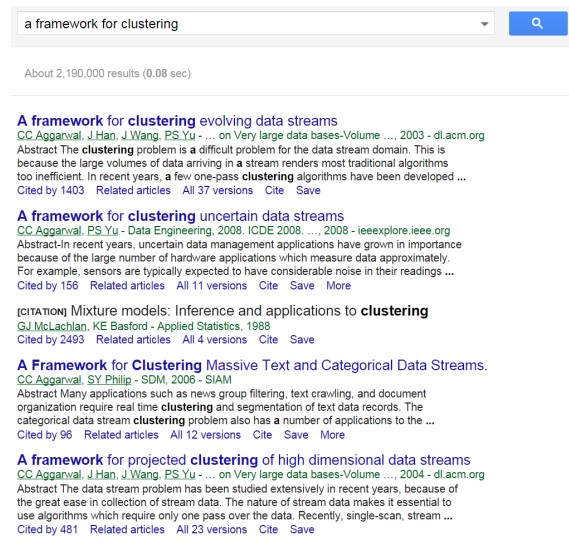

(c)

Fig. 5. Example of the search results for the query (a) 'clustering' (b) 'clustering in sensor networks' and (c) 'a framework for clustering'

method extracted important phrases as trigrams based on a procedure consisting of four processing steps. The trigrams extracted by the proposed method were evaluated as additional features in the paper title classification task using SVM. The experimental results showed that the accuracy was improved. We also compared the trigrams given by the proposed method with those given by the frequency-based method. According to the experimental results, the proposed method could provide as good trigrams as the frequency-based method. However, the proposed method has an extra gain, i.e., the bidirectional nature of trigrams extraction, which cannot be achieved by the frequency-based method. Further, we discussed how we could use such trigrams for query expansion. A search system using this type of query expansion can give search results relating to specific topics.

We have a future plan to perform a quantitative evaluation of the search results obtained by the query expansion based on the proposed method in information retrieval task.

\section{ACKNOWLEDGMENT}

This work has been supported by the Grant-in-Aid for enhancement of engineering higher education of the Japan International Cooperation Agency (JICA) from 2014 to 2017. We are grateful for their support.

\section{REFERENCES}

[1] K.S. Hasan and V. Ng, "Conundrums in unsupervised keyphrase extraction: making sense of the start-of-the-art," in Proc. of the 23rd International Conference on COLING 2010, Beijing, pp. 365-373, August 2010.

[2] C. Caragea, F. Bulgarov, A. Godea, S.D. Gollapalli, "Citation-enhanced keyphrase extraction from research papers: a supervised approach," in Proc. of the 2014 Conference on Empirical Methods in Natural Language Processing (EMNLP), pp. 1435-1446, Doha, Qatar, October 2014.

[3] R. Mihalcea, P. Tarau and E. Figa, "PageRank on semantic networks with application to word sense disambiguation," in Proc. of the 20th International Conference on Computational Linguistics, Stroudsburg, PA, USA, no. 1126, August 2004.

[4] S.D. Gollapalli and C. Caragea, "Extracting keyphrases from research papers using citation networks," in Proc. of the 28th AAAI Conference on Artificial Intelligence, pp. 1629-1635, June 2014.

[5] S. Choi, S. Cha, and C.C. Tappert, "A survery of binary similarity and distance measures," J.Syst. Cybern, Inf., vol. 8, no. 1, pp. 43-48, 2010.
[6] D. X. Wang, X. Gao, and P. Andreae, "Automatic keyword extraction from single-sentence natural language queries," in Proc. of the 12th Pacific Rim International Conference on Artificial Intelligence, Kuching, Malaysia, pp. 637-648, September 2012.

[7] K. Zhang, H. Xu, J. Tang, and J. Li, "Keyword extraction using support vector machine," in Proc. of the 7th International Conference on WAIM, Hong Kong, China, pp. 85-96, June 2006.

[8] T. Nomoto and Y. Matsumoto, "A new approach to unsupervised text summarization," in Proc. of SIGIR2001, pp. 26-34, 2001.

[9] M. R. Amini and P. Gallinari, "The use of unlabeled data to improve supervised learning for text summarization," in Proc. of SIGIR2002, 105-112, 2002

[10] S. N. Kim and M. Kan, "Re-examining automatic keyphrase extraction approaches in scientific articles," in Proc. of 2009 Workshop on Multiword Expressions, ACL-IJCNLP 2009, pp. 9-16, Suntec, Singapore, August 2009.

[11] Z. Liu, P. Li, Y. Zheng and M. Sun, "Clustering to find exemplar terms for keyphrase extraction," in Proc. of 2009 Conference on Empirical Methods in Natural Language Processing, pp. 257-266, Singapore, August 2009.

[12] T. Tomokiyo and M. Hurst, "A language model approach to keyphrase extraction," in Proc. of ACL 2003 Workshop on Multiword Expressions: Analysis, Acquisition and Treatment, vol. 18, pp. 33-40, 2003.

[13] K. Barker and N. Cornacchia, "Using noun phrase heads to extract document keyphrases," in Proc. of 13th Biennial Conference of the Canadian Society for Computational Studies of Intelligence, pp. 4052, May 2000.

[14] S. N. Kim, T. Baldwin and M. Kan, "Evaluating n-gram based evaluation metrics for automatic keyphrase extraction," in Proc. of $23 r d$ international conference on COLING 2010, pp. 572-580, Beijing, August 2010.

[15] R. Mihalcea, "Graph-based ranking algorithms for sentence extraction, applied to text Summarization," in Proc. of ACL 2004 on Interactive Poster and Demonstration Sessions, Article no. 20, Stroudsburg, PA, USA, July 2004

[16] X. Wan and J. Xiao, "CollabRank: towards a collaborative approach to single-document keyphrase extraction," in Proc. of 22nd International Conference on COLING 2008, pp. 969-976, Manchester, August 2008.

[17] M. Litvak and M. Last, "Graph-based keyword extraction for singledocument summarization," in Proc. of 08 MMIES on COLING 2008, pp. 17-24, Manchester, August 2008.

[18] Y. Win and T. Masada, "Exploring technical phrase frames from research paper titles," in Proc. of 29th IEEE International Conference on WAINA-2015, pp. 558-563, Gwangju, South Korea, 2015. 